University of Nebraska - Lincoln

DigitalCommons@University of Nebraska - Lincoln

Faculty Publications from the Department of Electrical \& Computer Engineering, Department Electrical and Computer Engineering

2010

\title{
Interface polarization coupling in piezoelectric-semiconductor ferroelectric heterostructures
}

\author{
Venkata M. Voora \\ University of Nebraska-Lincoln \\ Tino Hofmann \\ University of Nebraska-Lincoln, thofmann4@unl.edu \\ M. Brandt \\ Institut für Experimentelle Physik II, Universität Leipzig, 04103 Leipzig, Germany \\ M. Lorenz \\ Institut für Experimentelle Physik II, Universität Leipzig, 04103 Leipzig, Germany \\ M. Grundmann \\ Institut für Experimentelle Physik II, Universität Leipzig, 04103 Leipzig, Germany \\ See next page for additional authors
}

Follow this and additional works at: https://digitalcommons.unl.edu/electricalengineeringfacpub

Part of the Electrical and Computer Engineering Commons

Voora, Venkata M.; Hofmann, Tino; Brandt, M.; Lorenz, M.; Grundmann, M.; Ashkenov, N.; Schmidt, H.; Ianno, Natale J.; and Schubert, Mathias, "Interface polarization coupling in piezoelectric-semiconductor ferroelectric heterostructures" (2010). Faculty Publications from the Department of Electrical and Computer Engineering. 110.

https://digitalcommons.unl.edu/electricalengineeringfacpub/110

This Article is brought to you for free and open access by the Electrical \& Computer Engineering, Department of at DigitalCommons@University of Nebraska - Lincoln. It has been accepted for inclusion in Faculty Publications from the Department of Electrical and Computer Engineering by an authorized administrator of DigitalCommons@University of Nebraska - Lincoln. 


\section{Authors}

Venkata M. Voora, Tino Hofmann, M. Brandt, M. Lorenz, M. Grundmann, N. Ashkenov, H. Schmidt, Natale J. Ianno, and Mathias Schubert 


\title{
Interface polarization coupling in piezoelectric-semiconductor ferroelectric heterostructures
}

\author{
Venkata M. Voora, ${ }^{1}$ T. Hofmann, ${ }^{1}$ M. Brandt, ${ }^{2}$ M. Lorenz, ${ }^{2}$ M. Grundmann, ${ }^{2}$ N. Ashkenov, ${ }^{2}$ H. Schmidt, ${ }^{3}$ \\ N. Ianno, ${ }^{1}$ and M. Schubert ${ }^{1}$ \\ ${ }^{1}$ Department of Electrical Engineering and Nebraska Center for Materials and Nanoscience, University of Nebraska-Lincoln, \\ Lincoln, Nebraska 68588, USA \\ ${ }^{2}$ Institut für Experimentelle Physik II, Universität Leipzig, 04103 Leipzig, Germany \\ ${ }^{3}$ Institut für Ionenstrahlphysik und Materialforschung, Forschungszentrum Dresden-Rossendorf e.V., 01328 Dresden, Germany
}

(Received 18 November 2009; revised manuscript received 28 March 2010; published 5 May 2010)

\begin{abstract}
We present a dielectric continuum model approach for studying the electrical polarization properties of interface polarization coupled $\mathrm{BaTiO}_{3}, \mathrm{BaTiO}_{3}-\mathrm{ZnO}$, and $\mathrm{ZnO}-\mathrm{BaTiO}_{3}-\mathrm{ZnO}$ thin-film structures consisting of several hundred nanometer thick layers. Our model augments the effects of electric field driven switchable polarization and depletion layer formation with spontaneous interface polarization coupling. Wurtzite-structure (piezoelectric) $n$-type $\mathrm{ZnO}$ and perovskite-structure (ferroelectric) highly insulating $\mathrm{BaTiO}_{3}$ layers were prepared and investigated. The coupling between the nonswitchable spontaneous polarization of $\mathrm{ZnO}$ and the electrically switchable spontaneous polarization of $\mathrm{BaTiO}_{3}$ causes strong asymmetric polarization hysteresis behavior. The $n$-type $\mathrm{ZnO}$ reveals hysteresis-dependent capacitance variations upon formation of depletion layers at the $\mathrm{ZnO} / \mathrm{BTO}$ interfaces. We obtain a very good agreement between our model generated data and our experiment. Our model approach allows for derivation of the amount and orientation of the spontaneous polarization of the piezoelectric constituents and can be generalized toward multiple-layer piezoelectricsemiconductor ferroelectric heterostructures. We identify interface polarization coupled triple-layer ZnO-BTO$\mathrm{ZnO}$ heterostructures as two-terminal unipolar ferroelectric Bi-junction transistor for use in memory storage.
\end{abstract}

DOI: 10.1103/PhysRevB.81.195307

PACS number(s): 77.80.Fm, 77.80.Dj, 73.40.Qv, 81.05.Dz

\section{INTRODUCTION}

The physics and device characteristics of metalferroelectric-metal (MFM), ferroelectric-semiconductor (FS), metal-ferroelectric-semiconductor (MFS), and semiconductor-ferroelectric-semiconductor (SFS) heterostructures continue to attract attention due to their large potential for electronic and optoelectronic device applications. ${ }^{1-11}$ The ferroelectric constituent possesses switchable dielectric polarization. This polarization can be exploited for modification of the electronic and optical properties of a semiconductor heterostructure. Hysteresis properties of the ferroelectric polarization allows for bistable interface polarization configuration and potentially for bistable heterostructure operation modes. In this paper, we discuss the incorporation of piezoelectric semiconductors with spontaneous polarization in combination with ferroelectric materials. We anticipate coupling phenomena between the spontaneous polarizations at their common interface. ${ }^{12}$ Such configurations are of interest for bistable history-dependent heterostructure operation modes, such as for low-energy consumptive nonvolatile memory devices or ferroelectric field-effect transistors. ${ }^{13,14}$ MFS heterostructures typically reveal voltage-asymmetric polarization response. ${ }^{15-22} \mathrm{Xu}$ et al. ${ }^{15}$ suggested a model based on energy-band theory to explain the asymmetric behavior observed in lead zirconate titanate, barium titanate, strontium niobate, and lead barium niobate thin films deposited on silicon. Wurfel et al. ${ }^{17,18}$ and Batra et $a l .{ }^{19}$ considered ferroelectric depolarization and interface depletion layer formation for MFS and SFS structures, where depolarization charges are responsible for the decay of the ferroelectric polarization, and depletion formation occurs due to the band bending at the semiconductor-ferroelectric inter- face. Berman solved the Poisson equation for MFS structures in analogy to MFM structures with symmetric ferroelectric hysteresis properties and bound interface charges and successfully described the observed asymmetric hysteresis properties. ${ }^{16,20}$

As discussed in this work, for materials with appropriate electronic configurations, the net component of the interface charge is associated with internal electric fields, which can be used to modify or create space charge regions on either side of the interface. The semiconductor and the piezoelectric constituent may be comprised within the same material. Such heterostructures can be composed of, for example, from piezoelectric semiconducting wurtzite-structure $\mathrm{ZnO}$ and ferroelectric highly insulating perovskite-structure $\mathrm{BaTiO}_{3}$ (BTO). Solid-state materials with lack of symmetry inversion possess piezoelectric (stress- or strain-induced) polarization. Wurtzite-structure materials exhibit in addition to stress- or strain-induced polarization a spontaneous polarization component, which arises from the molecular arrangement within the unit cell. In equilibrium condition, a permanent static electric dipole forms along the lattice $c$ axis, with orientation and magnitude depending on the elemental lattice constituents. ${ }^{23-25}$ The amount of the spontaneous polarization depends on the elemental constitution within the wurtzite lattice and can be changed, for example, by alloying. ${ }^{26,27}$ The interface polarization coupling in piezoelectricsemiconductor ferroelectric heterostructures produces voltage-asymmetric hysteresis-dependent internal electric field variations, which affect their capacitance properties. In this paper, we introduce $n$-type conductive $\mathrm{ZnO}$ as the piezoelectric semiconducting constituent. The approach lined out is sufficiently general and also allows for incorporation of $p$-type conductive materials, as well as other piezoelectric semiconductors than $\mathrm{ZnO}$. BTO is incorporated as the ferro- 
electric constituent. We present a dielectric continuum model approach. We employ this approach for predicting and discussing the electrical polarization properties of interface polarization coupled $\mathrm{BaTiO}_{3}, \quad \mathrm{BaTiO}_{3}-\mathrm{ZnO}$, and $\mathrm{ZnO}-\mathrm{BaTiO}_{3}-\mathrm{ZnO}$ thin-film heterostructures. We augment known ferroelectric polarization model approaches with the phenomenon of electric-field and polarization-induced depletion layer formation. We predict and observe experimentally strong asymmetric polarization hysteresis behavior due to the $n$-type conductivity in $\mathrm{ZnO}$, which produces ferroelectric hysteresis-dependent electrical capacitance variations upon formation of depletion zones at the $\mathrm{ZnO}$-BTO interfaces. We obtain a very good quantitative agreement between our model generated data and our experiment. Our model approach allows for derivation of the amount and orientation of the spontaneous polarization of the piezoelectric constituent and can be generalized toward multiple-layer piezoelectricsemiconductor ferroelectric heterostructures. A qualitative interpretation based on our model is further used to explain observed electrical conductance hysteresis in $\mathrm{BaTiO}_{3}-\mathrm{ZnO}$ and $\mathrm{ZnO}-\mathrm{BaTiO}_{3}-\mathrm{ZnO}$ thin-film heterostructures, with potential for use in memory storage applications, and ferroelectric field-effect transistors. ${ }^{13,14}$ We note that the typical layer thickness considered here is on the order of fractions of micrometers or few micrometers, where our dielectric continuum model is reasonably applicable. Strong contemporary interest exists both theoretical and experimental on monolayer or few-layer ferroelectric heterostructures, see, for example, Ref. 28. At the nanoscale, additional phenomena will occur, and the here presented model does not apply to "genuinely thin," i.e., few atomic layer structures instantly. We further note that mechanical constraints imposed by the substrate or by the lattice mismatch between heterostructure constituents are very important and may need to be considered since strain imposed during pseudomorphic or partially relaxed heteroepitaxial growth will add another interface polarization component. This piezoelectric polarization can easily be augmented as new variable within our model approach which needs to be added to the spontaneous polarization component with magnitude and direction depending on type and amount of built-in strain for each layer.

Knowledge of magnitude and orientation of the spontaneous polarization is not exhaustive for many piezoelectric materials with spontaneous electric lattice dipole. Even for materials with large spontaneous polarization, such as $\mathrm{ZnO}$, little knowledge has been gathered so far. Theoretical investigations have calculated the charge per atom and the lattice parameters of the material, resulting in values for the spontaneous polarization of $P_{\mathrm{ZnO}}=-0.032 \mathrm{C} / \mathrm{m}^{2},{ }^{23} \quad P_{\mathrm{ZnO}}=$ $-0.05 \mathrm{C} / \mathrm{m}^{2},{ }^{24}$ and $P_{\mathrm{ZnO}}=-0.057 \mathrm{C} / \mathrm{m}^{2}{ }^{25}$ The spread of the values obtained is larger in other studies, which rely on experimental values for the lattice constants. ${ }^{29,30}$ Initial experimental studies employing second harmonic generation reported $P_{\mathrm{ZnO}}=-0.07 \pm 0.015 \mathrm{C} / \mathrm{m}^{2}$. ${ }^{31}$ The influence of the $\mathrm{ZnO}$ polarization on the electronic structure and the formation of defects has been studied by photoluminescence spectroscopy, current-voltage, capacitance-voltage, and Halleffect investigations. ${ }^{32-37}$ The two different polar surfaces of $\mathrm{ZnO}$ ( $\mathrm{Zn}$ or $\mathrm{O}$ termination) have been investigated for their physical properties. The relative intensities of near-band-gap photoluminescence features were found different between the two polar surfaces, which was attributed to different surface Fermi-energy positions. ${ }^{34}$ It was reported that free charge carrier densities ${ }^{33,35-37}$ and surface chemistry mechanisms differ ${ }^{32}$ and a remarkable difference in the electronic work function was observed. ${ }^{33}$ Different surface reconstructions were reported recently. ${ }^{38}$ The polarity of a $\mathrm{ZnO}$ single crystal can be determined by etching a polar surface. ${ }^{39}$ The polarity was also studied in high-quality crystalline $\mathrm{ZnO}$ thin films by dispersion $\mathrm{x}$-ray diffraction, ${ }^{40-42}$ scanning nonlinear dielectric microscopy, ${ }^{43}$ angular resolved x-ray photoelectron diffraction, ${ }^{44,45}$ and coaxial-impact collision-ion scattering spectroscopy. ${ }^{46-48}$ Detailed studies of the spontaneous polarization properties have been carried out in the structurally equivalent AlGaN-GaN material system. ${ }^{26,27}$ At heterostructure interfaces within the AlGaN system, in addition to the spontaneous polarization component a large compressive or tensile strain-induced polarization exists. The dependence of both polarizations on the $\mathrm{Al}$ concentration, for example, in AlGaN-GaN, can be studied by investigating the electronic properties of two-dimensional carrier gases, which can form at the interface. Similar investigations have been carried out in the $\mathrm{MgZnO}-\mathrm{ZnO}$ system ${ }^{49-51}$ where, however, the latticemismatch induced strain is smaller than in the AlGaN-GaN system, and a cubic (rocksalt-structure) phase transition occurs in $\mathrm{Zn}_{x} \mathrm{Mg}_{1-x} \mathrm{O}$ for $x \approx 0.65 .^{52}$ Experimental polarization values have been obtained by numerical evaluation of the photoluminescence shift in $\mathrm{MgZnO}-\mathrm{ZnO}$ quantum well structures. The polarization-induced electric field within the quantum wells affects the optoelectronic properties by band bending and field-induced emission wavelength shift (Stark-effect). ${ }^{53}$ The obtained values represent the net polarization of the heterostructure constituents, both possessing piezoelectric and spontaneous polarization, and were found to vary widely among different samples and also from theoretical predictions. ${ }^{23}$ The decomposition of the net polarization into strain-induced and piezoelectric components requires knowledge of lattice constants and piezoelectric coefficients.

We have recently reported on electro-optic and electric investigations of ZnO-BTO heterostructures, ${ }^{12,54-59}$ where we observed asymmetric polarization properties. Our model approach was briefly mentioned in two previous proceedings publications. ${ }^{57,60}$ In this work, we present our model approach in detail and give a quantitative comparison with experimental data. Section II details the model approach. Section III discusses experimental procedures and sample details. Section IV presents results and discussions. Conclusions are summarized in Sec. V. Appendix A recalls the phenomenological ferroelectric polarization approximation employed here. Appendix B describes the experimental polarization measurement technique (Sawyer-Tower circuit), and the employed numerical iteration procedure is outlined in Appendix C.

\section{THEORY}

Figure 1 depicts a schematic presentation of a piezoelectric semiconductor $(\mathrm{P})$-ferroelectric $(\mathrm{F})$-piezoelectric semi- 


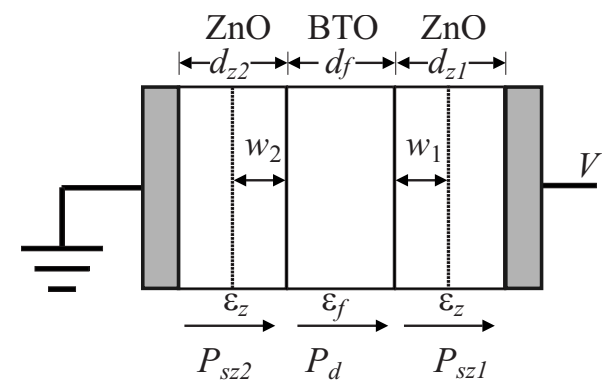

FIG. 1. Schematic of a piezoelectric semiconductor (P)ferroelectric (F)-piezoelectric semiconductor (P) heterostructure (PFP) with spontaneous piezoelectric and ferroelectric polarization $P_{\text {sz }}$ and $P_{\mathrm{d}}$, respectively, embedded between ideal contacts. Components for $\mathrm{P}$ and $\mathrm{F}$ are here represented by $\mathrm{ZnO}$ and $\mathrm{BaTiO}_{3}$ (BTO), respectively, with symmetric layer sequence. The left interface of the left layer is connected to ground, the right interface of the right layer is at potential $V$. Depletion layer formation occurs at interfaces (from left to right) P-F $\left(w_{2}\right)$ and/or F-P $\left(w_{1}\right)$ for $n$-type (conductive) constituents $\mathrm{P}$ and insulating (barrier) F. $w_{1}, w_{2}$ depend on $V$, sample properties $\left(P_{\mathrm{sz} 1}, P_{\mathrm{sz} 2}\right.$, layer thickness $d_{\mathrm{z} 1}, d_{\mathrm{z} 2}, d_{\mathrm{f}}$, dielectric constants $\left.\varepsilon_{\mathrm{z}}, \varepsilon_{\mathrm{f}}\right)$, and sample history $\left(P_{\mathrm{d}}\right)$.

conductor (P) heterostructure (PFP) embedded between ideal contacts. The biased and grounded $\mathrm{P}$ layers $(\mathrm{ZnO})$ have layer thickness $d_{\mathrm{z} 1}$ and $d_{\mathrm{z} 2}$, respectively, and layer $\mathrm{F}$ (BTO) has thickness $d_{\mathrm{f}}$. Layer $\mathrm{F}$ is considered ideally as an insulator but more realistically here as a highly resistive dielectric layer and which acts as an electronic barrier. Layers $\mathrm{P}$ are treated as $n$-type semiconductors with equal net dopant concentration $N_{\mathrm{c} 1}$ (biased layer P) and $N_{\mathrm{c} 2}$ (grounded layer P). The nonswitchable spontaneous polarization in layers $\mathrm{P}$ causes a permanent electric field and biases the switching behavior of the ferroelectric polarization in layer F. Both polarizations influence the formation of space charge regions, such as depletion or accumulation layers within layer $\mathrm{P}$ toward its interface with layer F. Two possible polar (crystallographic) orientations for $\mathrm{P}$ give rise to two possible spontaneous polarization vector orientations $P_{\mathrm{sz}}$. In triple or multiple layer heterostructures, polarization coupling can occur at multiple interfaces ${ }^{61-67}$ and will become interdependent. The charge distribution at the interfaces will affect the electrical properties of the overall heterostructure. For the triple layer structure, two scenarios can be envisioned, where either layer $\mathrm{F}$ is embedded by layers $\mathrm{P}$ or vice versa. In this section, we present a model describing a PFP heterostructure. The model equations for the situation FPF are obtained in straightforward manner using the scheme discussed below.

In a PFP heterostructure, at each of the P-F and F-P interfaces, depletion or accumulation layers can be formed toward each interface within each layer $\mathrm{P}$, depending on $\mathrm{P}$ and F materials' properties, history, and external bias. Upon depletion layer formation, a capacitive sublayer is formed in layer P toward layer F, which augments a series capacitance in addition to layer F. Assuming homogeneous distribution of net donor density $N_{\mathrm{c}}$ within layer P of thickness $d$, a charge depletion layer with thickness $w$ causes a potential step

$$
V_{\text {depletion }}=\frac{e N_{\mathrm{c}}}{\varepsilon} \int_{0}^{w \leq d} x d x,
$$

where $\varepsilon$ is the dielectric constant in $\mathrm{P}, e$ is the electronic unit charge, and integration occurs along spatial variable $x$ parallel to the electric field. The sources of the electric field within the depletion layer are the ionized immobile donors, which may be partially screened by the net polarization charge at the P-F interface. The remaining nondepleted sublayer $d-w$ of $\mathrm{P}$ is here considered ideally conductive, i.e., with negligible series resistance (flatband situation). Accordingly, accumulation layer formation is omitted since no potential drop can occur across any nondepleted part of layer P. The path integral over the internal electric field between the two electrodes in Fig. 1 delivers

$$
V=d_{\mathrm{f}} E_{\mathrm{f}}+\int_{0}^{w_{1}} E_{\mathrm{z} 1} d x+\int_{0}^{w_{2}} E_{\mathrm{z} 2} d x
$$

which depends on the electric field across layer F, $E_{\mathrm{f}} . E_{\mathrm{z} 1}$ and $E_{\mathrm{z} 2}$ are the fields in layers $\mathrm{P}$ to the right and left of layer $\mathrm{F}$, respectively. $E_{\mathrm{f}}$ can be expressed by $P_{\mathrm{d}}, D_{\mathrm{f}}$, and $\varepsilon_{\mathrm{f}}$, the spontaneous (ferroelectric) polarization, displacement, and dielectric constant of layer F, respectively,

$$
E_{\mathrm{f}}=\varepsilon_{\mathrm{f}}^{-1}\left(D_{\mathrm{f}}-P_{\mathrm{d}}\right) .
$$

Note that $P_{\mathrm{d}}$ is a function of $E_{\mathrm{f}}$. With $D_{\mathrm{f}}=\sigma_{\mathrm{b}}$, the surface charge at the bottom of layer F, and Eq. (1), we obtain

$$
V=\frac{d_{\mathrm{f}}}{\varepsilon_{\mathrm{f}}}\left(\sigma_{\mathrm{b}}-P_{\mathrm{d}}\right)+\frac{1}{2}\left(\frac{e N_{\mathrm{c} 1}}{\varepsilon_{\mathrm{z}}} w_{1}^{2}-\frac{e N_{\mathrm{c} 2}}{\varepsilon_{\mathrm{z}}} w_{2}^{2}\right) .
$$

For nonzero $E_{\mathrm{z} 2}$, it must point from right to left in Fig. 1 in order to form depletion, which results in negative potential due to layer $w_{2}$ in Eq. (4). Dielectric displacement continuity requirement (from left to right in Fig. 1) across interface P-F results in

$$
e N_{\mathrm{c} 1} w_{1}+P_{\mathrm{sz} 1}=E_{\mathrm{f}} \varepsilon_{\mathrm{f}}+P_{\mathrm{d}},
$$

and across F-P

$$
E_{\mathrm{f}} \varepsilon_{\mathrm{f}}+P_{\mathrm{d}}=-e N_{\mathrm{c} 2} w_{2}+P_{\mathrm{sz} 2} .
$$

$P_{\mathrm{sz} 1}$ and $P_{\mathrm{sz} 2}$ are the spontaneous (piezoelectric) polarizations of layers $\mathrm{P}$ and $\varepsilon_{\mathrm{z}}$ is the dielectric constant of layers $\mathrm{P}$. Equations (4)-(6) connect quantities $\sigma_{\mathrm{b}}, E_{\mathrm{f}}, w_{1}$, and $w_{2}$. Note that we assume no spontaneous interface charge at the depleted-nondepleted interface within layer $\mathrm{P}$, consistent with the above made "flatband" assumption.

Variation in orientation and magnitude of polarization in layers $\mathrm{P}$ results in varieties of possible arrangements. For example, assuming for simplicity $\left|P_{\mathrm{sz} 1}\right|=\left|P_{\mathrm{sz} 2}\right|$, one can have $P_{\mathrm{sz} 1}=P_{\mathrm{sz} 2}=P_{\mathrm{sz}}$, i.e., $\mathrm{P}^{+} \mathrm{FP}^{+}$, or $P_{\mathrm{sz} 1}=-P_{\mathrm{sz} 2}=P_{\mathrm{sz}}$, i.e., $\mathrm{P}^{+} \mathrm{FP}^{-}$, or $\mathrm{P}^{-} \mathrm{FP}^{+}$, or $\mathrm{P}^{-} \mathrm{FP}^{-}$, accordingly. Depending on external bias $V$ and material parameter situations, different configurations of depletion layer formation can occur (Table I). Neither $w_{1}$ and $w_{2}$ can be negative or larger than $d_{\mathrm{z} 1}$ and $d_{\mathrm{z} 2}$. Scheme (A) is the nondepleted state of both layers P. Scheme (B) is when the biased layer $\mathrm{P}$ (right in Fig. 1) possesses a finite depletion layer $\left(0<w_{1}<d_{\mathrm{z} 1}\right)$, and the grounded layer $\mathrm{P}$ (left in Fig. 1) is nondepleted. Scheme (C) is when grounded 
TABLE I. Configuration schemes according to depletion layer formation, variables $w_{1}, w_{2}$ in Eq. (10), and corresponding junction (Jctn) conditions (forwardlike "F," reverselike "R;" see Sec. IV B for definition of $\mathrm{F}$ and $\mathrm{R}$ ) between piezoelectric and ferroelectric $(\mathrm{P}-\mathrm{F})$, and ferroelectric and piezoelectric (F-P) constituents of the PFP heterostructure shown in Fig. 1.

\begin{tabular}{lcccc}
\hline \hline Scheme & $w_{1}$ & $w_{2}$ & Jctn P-F & Jctn F-P \\
\hline (A) & 0 & 0 & $\mathrm{~F}$ & $\mathrm{~F}$ \\
(B) & $0<w_{1}<d_{\mathrm{z} 1}$ & 0 & $\mathrm{R}$ & $\mathrm{F}$ \\
(C) & 0 & $0<w_{2}<d_{\mathrm{z} 2}$ & $\mathrm{~F}$ & $\mathrm{R}$ \\
(D) & $0<w_{1}<d_{\mathrm{z} 1}$ & $0<w_{2}<d_{\mathrm{z} 2}$ & $\mathrm{R}$ & $\mathrm{R}$ \\
(E) & $d_{\mathrm{z} 1}$ & 0 & $\mathrm{R}$ & $\mathrm{F}$ \\
(F) & 0 & $d_{\mathrm{z} 2}$ & $\mathrm{~F}$ & $\mathrm{R}$ \\
(G) & $0<w_{1}<d_{\mathrm{z} 1}$ & $d_{\mathrm{z} 2}$ & $\mathrm{R}$ & $\mathrm{R}$ \\
(H) & $d_{\mathrm{z} 1}$ & $0<w_{2}<d_{\mathrm{z} 2}$ & $\mathrm{R}$ & $\mathrm{R}$ \\
(I) & $d_{\mathrm{z} 1}$ & $d_{\mathrm{z} 2}$ & $\mathrm{R}$ & $\mathrm{R}$ \\
\hline \hline
\end{tabular}

layer $\mathrm{P}$ possesses a finite depletion layer $\left(0<w_{2}<d_{\mathrm{z} 2}\right)$, and biased layer $\mathrm{P}$ is nondepleted. In scheme (D) both biased and grounded layers $\mathrm{P}$ are partially depleted $\left(0<w_{1}<d_{\mathrm{z} 1}, 0\right.$ $<w_{2}<d_{\mathrm{z} 2}$ ). In scheme (E) the biased and grounded layers $\mathrm{P}$ are fully and nondepleted, i.e., $w_{1}=d_{\mathrm{z} 1}, w_{2}=0$, respectively. Scheme $(\mathrm{F})$ is the reverse case of $(\mathrm{E})$, i.e., $w_{1}=0, w_{2}=d_{\mathrm{z} 2}$. Further conceivable schemes are $(\mathrm{G})$ and $(\mathrm{H})$ similar to $(\mathrm{B})$ and $(\mathrm{C})$, except with $w_{2}=d_{\mathrm{z} 2}$ and $w_{1}=d_{\mathrm{z} 1}$, respectively, and scheme (I) with $w_{1}=d_{\mathrm{z} 1}, w_{2}=d_{\mathrm{z} 2}$. Note that for schemes (E)(I), the space-charge related displacement terms $e N_{\mathrm{c} 1} d_{\mathrm{z}}$ and/or $-e N_{\mathrm{c} 2} d_{\mathrm{z}}$ are constant, and additional potential $E_{\mathrm{z}} d_{\mathrm{z}}$ and displacement $E_{\mathrm{z}} \varepsilon_{\mathrm{z}}$ terms must be added to the appropriate sides of Eqs. (4)-(6), respectively.

In our model approach we aim at comparison with experimentally observable time-dependent electric potential variations, i.e., Sawyer-Tower (ST) measurements (Appendix B). ST measurements monitor $d / d t(V)=d / d t\left(V_{\text {in }}-V_{\mathrm{o}}-V_{\mathrm{bi}}\right)$ across our heterostructure relative to a normal (standard) capacitor structure, where $V_{\text {in }}$ is the applied bias to the series of sample and normal capacitor, $V_{\mathrm{o}}$ is the potential drop across the normal capacitor, and $V_{\mathrm{bi}}$ is the effective built-in potential (sum of all conduction band offsets) across the sample. The ohmic series resistance $R_{\mathrm{s}}$ of the sample between ground and bias contact causes parasitic current. $R_{\mathrm{s}}$ is dominated by the highly resistive layer F. $R_{\mathrm{s}}$ is measured by experiment and considered here as a constant. ST measurements are commonly employed for dielectric hysteresis measurements of ferroelectric capacitor structures. We are here interested in calculation of time derivatives of the charge leaving or entering ground. Because all layers are in series and for continuous time-harmonic excitation $d V / d t$, in steady-state condition the charge variation at the ground contact equals $d \sigma_{\mathrm{b}} / d t$. Taking the time derivative of Eqs. (4)-(6) and substituting differentials

$$
d P_{\mathrm{d}}=\left(\frac{d P_{\mathrm{d}}}{d t}\right)\left(\frac{d E_{\mathrm{f}}}{d t}\right)^{-1} d E_{\mathrm{f}},
$$

we obtain linear relations between $d E_{\mathrm{f}} / d t$ and $d \sigma_{\mathrm{b}} / d t$, with the time derivative of the external potential

$$
\frac{d E_{\mathrm{f}}}{d t}=\gamma \frac{d V}{d t}
$$

and

$$
\frac{d \sigma_{\mathrm{b}}}{d t}=\gamma\left(\varepsilon_{\mathrm{f}}+\frac{d P_{\mathrm{d}}}{d E_{\mathrm{f}}}\right) \frac{d V}{d t},
$$

with

$$
\gamma=\left(d_{\mathrm{f}}+\frac{w_{1}+w_{2}}{\varepsilon_{\mathrm{z}}}\left[\varepsilon_{\mathrm{f}}+\frac{d P_{\mathrm{d}}}{d E_{\mathrm{f}}}\right]\right)^{-1} .
$$

The coefficient $\gamma$ differs for the different schemes by the values of the depletion layer variables $w_{1}$ and $w_{2}$ (Table I). Without hysteresis in $\frac{d P_{\mathrm{d}}}{d E_{\mathrm{f}}}$, Eqs. (8) and (9) become simple linear relationships.

The ferroelectric nature of layer $\mathrm{F}$ causes nonlinear dependence $P_{\mathrm{d}}\left(E_{\mathrm{f}}\right)$, which renders the ferroelectric hysteresis. $P_{\mathrm{d}}\left(E_{\mathrm{f}}\right)$ is an intricate function of microstructure, defects, and time. The hyperbolic tangent function ${ }^{2}$ is used here as approximation for the saturation polarization $P_{\text {sat }}$ of layer $\mathrm{F}$

$$
P_{\text {sat }}^{+}\left(E_{\mathrm{f}}\right)=P_{\mathrm{s}} \tanh \left[\frac{E_{\mathrm{f}}-E_{\mathrm{c}}}{2 \delta}\right],
$$

for ascending $E_{\mathrm{f}}$, and

$$
P_{\text {sat }}^{-}\left(E_{\mathrm{f}}\right)=-P_{\text {sat }}^{+}\left(-E_{\mathrm{f}}\right),
$$

for the descending branch, where $P_{\mathrm{s}}, E_{\mathrm{c}}$, and $\delta$ are the saturation polarization, coercive field, and idealized ferroelectric domain distribution parameter $(\delta$ being small or large for large or small domains representing domain interaction, respectively) (Appendix A). In order to use the above expressions for meaningful model calculations of experimental data, we implement numerical iteration schemes due to the nonlinear and hysteresis behavior of function $P_{\mathrm{d}}\left(E_{\mathrm{f}}\right)$. In particular, at given external potential $V, P_{\mathrm{d}}\left(E_{\mathrm{f}}\right)$ is expanded to first order in $E_{\mathrm{f}}$, where the derivative is obtained analytically from differentiation of Eqs. (11) and (12) (Appendix A), and evaluated at given $E_{\mathrm{f}} . E_{\mathrm{f}}, w_{1}$, and $w_{2}$ are then calculated using Eqs. (4)-(6) in linear expansion for $P_{\mathrm{d}}\left(E_{\mathrm{f}}\right)$. Appendix $\mathrm{C}$ summarizes the linear expansions. The pivoting parameters for the selection of the calculation scheme in this iteration approach are $w_{1}$ and $w_{2}$. Depending on whether $w_{1}$ and $w_{2}$ are both or individually negative, zero, positive, or larger than $d_{\mathrm{z} 1}$ and $d_{\mathrm{z} 2}$, respectively, the corresponding scheme of (A) -(I) is selected. For negative $w_{1}$ and/or $w_{2}$, the respective value(s) is (are) set to zero. Likewise, for $w_{1}$ and/or $w_{2}$ larger than $d_{\mathrm{z} 1}$ and/or $d_{\mathrm{z} 2}$, the corresponding value(s) is (are) set to $d_{\mathrm{z} 1}$ and/or $d_{\mathrm{z} 2}$, respectively. Calculation of $E_{\mathrm{f}}, P_{\mathrm{d}}, w_{1}$, and $w_{2}$ is repeated until convergence for all varied parameters is obtained. For calculations, reasonable but otherwise arbitrary initial parameter settings are used. Typically, after few iterations, $E_{\mathrm{f}}, P_{\mathrm{d}}, w_{1}$, and $w_{2}$ converge to a stable solution. As a result, the internal electric field variation within the structure is obtained, which allows for calculation of the heterostructure response within the ST circuit. Furthermore, the variation in $P_{\mathrm{d}}$ versus $V_{\text {in }}$ is also obtained, which unravels the hysteresis functionality of layer $\mathrm{F}$ within the PFP heterostruc- 
ture. This functionality will be discussed together with the $\mathrm{ST}$ response in Sec. IV.

\section{EXPERIMENT}

\section{A. Sample preparation}

The BTO and ZnO layers were deposited by PLD, successively without breaking the vacuum. The substrate temperature and the background pressure while growing these materials were $700{ }^{\circ} \mathrm{C}$ and 0.06 mbar, respectively. The number of laser pulses was $10^{5}$ for BTO and $2 \times 10^{4}$ for $\mathrm{ZnO}$. A pulse energy of $600 \mathrm{~mJ}$ was used. The BTO and $\mathrm{ZnO}$ layers were highly textured polycrystalline [BTO with (111) and $\mathrm{ZnO}$ with (0001) texture]. The metal electrodes were grown by sputtering Pt ohmic contacts. Structural, optical, and electrical analysis were performed as described previously. ${ }^{12,54-59}$ The $\mathrm{ZnO}$ layers are $n$-type conductive, with free electron concentration $N_{\mathrm{c}}$ between $1 \times 10^{16}-1$ $\times 10^{17} \mathrm{~cm}^{-3}$. Net donor densities were determined by electrical Hall effect and Infrared Ellipsometry characterization. ${ }^{52}$ The BTO layer is highly resistive. PtBTO-Pt reference structures were prepared in the same PLD system.

\section{B. Polarization measurements}

All samples including the Pt-BTO-Pt reference structures were investigated by ST measurements (Appendix B) with varying amplitudes $\left(V_{\mathrm{P}-\mathrm{P}}\right)$ of sinusoidal sweep voltage at frequency $f$. The electrical polarization of the structure was monitored with reference to a normal (hysteresis free) capacitor $\left(C_{\mathrm{st}}=1 \mu \mathrm{F}\right)$. An external voltage $V_{\text {in }}$ is applied in series to the sample and $C_{\mathrm{st}}$. The potential variation $V_{\mathrm{o}}$ across $C_{\text {st }}$ is monitored as a function of $V_{\text {in }}$. Hysteresis properties are measured by cyclic (time-harmonic) variations in $V_{\text {in }}$, which result in loop formation of $V_{\mathrm{o}}$ versus $V_{\text {in }}$.

\section{RESULTS AND DISCUSSIONS}

\section{A. Single-layer F: Model}

With $d_{\mathrm{z} 1}=d_{\mathrm{z} 2}=0$ and $P_{\mathrm{sz} 1}=P_{\mathrm{sz} 2}=0$ the single-layer ferroelectric capacitor situation, i.e., a single ferroelectric layer sandwiched between ideal capacitor plates, is retained as solution within our model approach. Detailed discussions and presentations are omitted for brevity. A model comparison with experimental ST data from a single-layer BTO structure is discussed in Sec. IV D. Note that the internal ferroelectric domain configuration is approximated here as a quasisingle domain with effective ferroelectric polarization properties by means of Eqs. (A1)-(A4).

\section{B. Double-layer PF: Model}

The double-layer situation is obtained by setting $P_{\mathrm{sz} 1}$ $=P_{\mathrm{sz}}, P_{\mathrm{sz} 2}=0$, and allowing for schemes $(\mathrm{A}),(\mathrm{B})$, and $(\mathrm{E})$ only. Figure 2 depicts calculated loops $V_{\mathrm{o}}$ versus $V_{\text {in }}$, and $P_{\mathrm{d}}$ versus $V_{\text {in }}$ for different values of $P_{\mathrm{sz}}$. Parameters used for calculation are summarized in Table II. In particular, the graphs in Fig. 2 depict the influence (existence and direction)

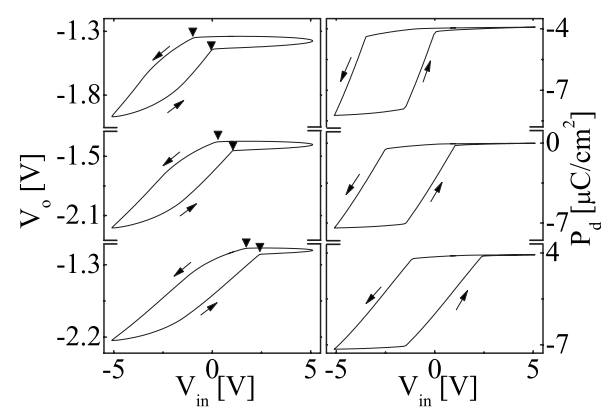

FIG. 2. Model calculated ST (left panel) and corresponding $P_{\mathrm{d}}$ traces versus $V_{\text {in }}$ (right panel) for different values of $P_{\mathrm{sz}}$ (traces top to bottom: $-4,0,4 \mu \mathrm{C} \mathrm{m}^{-2}$ ) for a double-layer PF structure. Arrows indicate the sweep direction $\left(V_{\mathrm{P}-\mathrm{P}}=5 \mathrm{~V}, f=1.5 \mathrm{kHz}\right)$. Solid triangles indicate transitions between schemes $(\mathrm{A})(w=0)$ and $(\mathrm{B})$ $\left(d_{\mathrm{z}}>w>0\right)$. Model parameters are listed in Table II.

of spontaneous polarization $P_{\mathrm{sz}}$ of layer $\mathrm{P}$ onto the electrical properties of the double-layer PF heterostructure. The heterostructure reveals a diodelike behavior. For negative bias $V_{\text {in }}$ the structure renders the forwardlike ("F" in Table I) bias characteristics of a semiconductor junction diode. Layer P is conductive, and the overall capacitance of the structure is

TABLE II. Best-match model calculation parameters for right and left layers $\mathrm{P}$ in Fig. 1 (thickness $d_{\mathrm{z} 1}=d_{\mathrm{z} 2}=d_{\mathrm{z}}$, spontaneous polarization $P_{\mathrm{sz}}$, net donor densities $N_{\mathrm{c} 1}, N_{\mathrm{c} 2}$, and dielectric constant $\varepsilon_{\mathrm{z}}$ ), layer $\mathrm{F}$ (thickness $d_{\mathrm{f}}$, remanent polarization $P_{\mathrm{r}}$, saturation polarization $P_{\mathrm{s}}$, coercive field $E_{\mathrm{c}}$, and dielectric constant $\varepsilon_{\mathrm{f}}$ ), conduction-band offset $V_{\mathrm{bi}}$ between $\mathrm{P}$ and F, ST parameters for normal capacitor $C_{\mathrm{st}}$, normal capacitor resistance $R_{\mathrm{n}}$, ST frequency $f$, and sample series resistance $R_{\mathrm{S}}$. All varied parameters are found with $1 \%$ uncertainty with standard $(90 \%)$ confidence interval.

\begin{tabular}{lccc}
\hline \hline & \multicolumn{3}{c}{ Best-match model values } \\
\cline { 2 - 4 } Parameter & BTO & BTO-ZnO & ZnO-BTO-ZnO \\
\hline$d_{\mathrm{z} 1}=d_{\mathrm{z} 2}(\mu \mathrm{m})$ & 0 & 0.5 & 0.5 \\
$d_{\mathrm{f}}(\mu \mathrm{m})$ & 1.5 & 1.45 & 1.4 \\
$N_{\mathrm{c} 1}\left(10^{17} \mathrm{~cm}^{-3}\right)$ & & 0.55 & 2.9 \\
$N_{\mathrm{c} 2}\left(10^{17} \mathrm{~cm}^{-3}\right)$ & & & 2 \\
$V_{\mathrm{bi}}(\mathrm{V})$ & 0 & $-0.78 \pm 0.2$ & 0 \\
$\varepsilon_{\mathrm{f}}$ & 202 & 250 & 80 \\
$\varepsilon_{\mathrm{z}}$ & & 8 & 6 \\
$R_{\mathrm{n}}(\mathrm{M} \Omega)^{\mathrm{a}}$ & 1 & 1 & 1 \\
$C_{\mathrm{n}}(\mu \mathrm{C})^{\mathrm{b}}$ & 1 & 1 & 1 \\
$E_{\mathrm{c}}\left(\mathrm{MV} / \mathrm{m}^{2}\right.$ & 1.15 & 1.1 & $1.1^{\mathrm{c}}$ \\
$P_{\mathrm{s}}\left(\mu \mathrm{C} \mathrm{cm}{ }^{-2}\right)$ & 7.54 & 14.1 & 6 \\
$P_{\mathrm{r}}\left(\mu \mathrm{C} \mathrm{cm}{ }^{-2}\right)$ & 1.39 & 5.6 & 0.11 \\
$f(\mathrm{kHz})^{\mathrm{d}}$ & 1.5 & 1.5 & 1.5 \\
$R_{\mathrm{s}}(\mathrm{k} \Omega)$ & 1000 & 13 & -0.1 \\
$P_{\mathrm{sz}}\left(\mu \mathrm{C} \mathrm{cm}{ }^{-2}\right)$ & & -4.1 & 1000 \\
\hline \hline
\end{tabular}

aParameter from measurement, not varied.

${ }^{\mathrm{b}}$ Parameter from measurement, not varied.

${ }^{\mathrm{c}} E_{\mathrm{c}}=1.1 \mathrm{MV} / \mathrm{m}\left(V_{\mathrm{P}-\mathrm{P}}=12 \mathrm{~V}\right) ; \quad E_{\mathrm{c}}=0.957 \mathrm{MV} / \mathrm{m}\left(V_{\mathrm{P}-\mathrm{P}}=10 \mathrm{~V}\right) ;$ $E_{\mathrm{c}}=0.781 \mathrm{MV} / \mathrm{m}\left(V_{\mathrm{P}-\mathrm{P}}=8 \mathrm{~V}\right) ; E_{\mathrm{c}}=0.622 \mathrm{MV} / \mathrm{m}\left(V_{\mathrm{P}-\mathrm{P}}=6 \mathrm{~V}\right)$.

${ }^{\mathrm{d}}$ Parameter from measurement, not varied. 


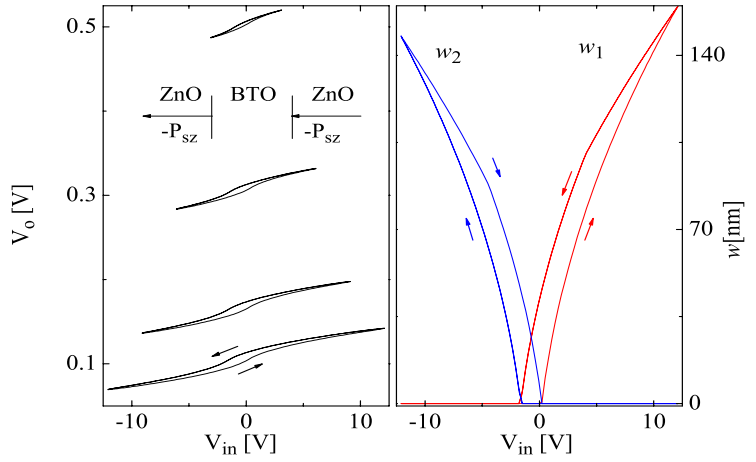

(a) $\mathrm{P}^{-} \mathrm{FP}^{-}$

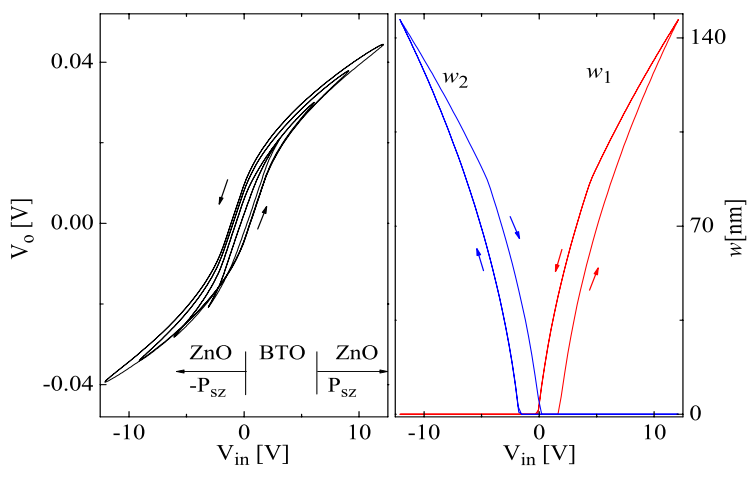

(c) $\mathrm{P}^{+} \mathrm{FP}^{-}$

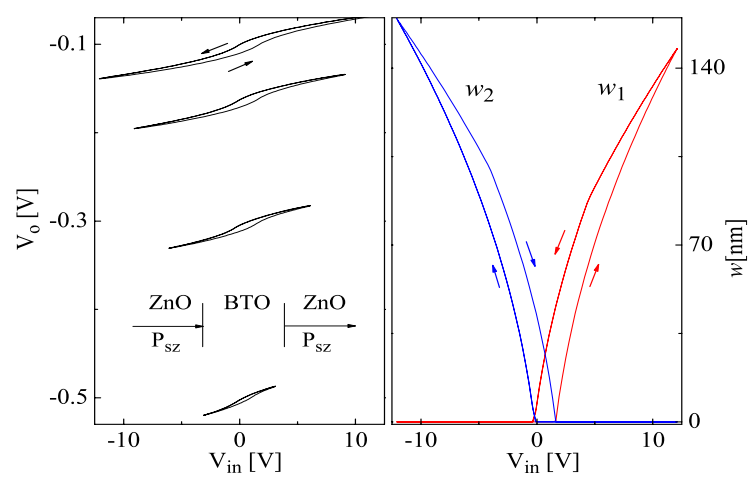

(b) $\mathrm{P}^{+} \mathrm{FP}^{+}$

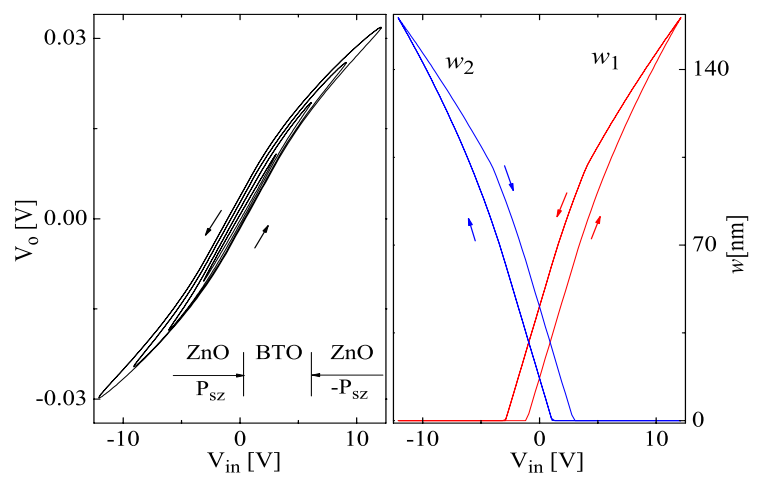

(d) $\mathrm{P}^{-} \mathrm{FP}^{+}$

FIG. 3. (Color online) Model calculated ST data [left panels in Figs. 3(a)-3(d); sweeping voltages $\left.V_{\mathrm{P}-\mathrm{P}}=3,6,9,12 \mathrm{~V}, f=1.5 \mathrm{kHz}\right]$ and $w_{1}, w_{2}$ [right panels in Figs. 3(a)-3(d); $V_{\mathrm{P}-\mathrm{P}}=12 \mathrm{~V}$ only] for triple-layer PFP heterostructure, and four different piezoelectric polarization vector orientations. Note that (a) and (b) correspond to simple contact reversal in Fig. 1, whereas (c) and (d) render two different physical structures. Arrows indicate voltage sweeping direction. Model parameters are those summarized in Table II for a ZnO-BTO-ZnO heterostructure, discussed in Sec. IV F.

established by layer F only. For positive external voltage, however, free electrons are gradually withdrawn from layer $\mathrm{P}$, forming a depletion layer $w>0$ with reverselike (" $\mathrm{R}$ " in Table I) bias characteristics thereby adding a small series capacitance to the overall sample. Hence, the slope of $V_{\mathrm{o}}$ versus $V_{\text {in }}$ decreases considerably since most of $V_{\text {in }}$ now drops across the sample, and not across the test capacitor. Within a series of capacitances, the overall capacitance is dominated by the small capacitance due to layer $w$. Thus the potential across layer $\mathrm{F}$ is small and $E_{\mathrm{f}}$ remains small, reflected by the nearly constant $P_{\mathrm{d}}$ (right panel of Fig. 2). In "forward" direction $(w=0)$, the electric field drops almost completely across the BTO layer and the ferroelectric polarization follows its typical hysteresis. In "reverse" direction, the ferroelectric polarization remains mostly within the negative part of the hysteresis loop. $P_{\mathrm{sz}}$ shifts the transition at which depletion layer formation occurs, i.e., the threshold between "reverse" and "forward" direction. The onset of the depletion layer formation and the associated capacitance change is shifted to negative $\left(P_{\mathrm{sz}}<0\right)$ or positive voltages $\left(P_{\mathrm{sz}}>0\right)$. The transitions where the depletion layer switches between zero and nonzero are indicated by vertical solid triangles in the left panel of Fig. 2. Note that in "reverse" direction, $P_{\mathrm{d}}$ approaches $P_{\mathrm{sz}}$. Due to the nonlinear functionality and hysteresis of $P_{\mathrm{d}}$ versus $E_{\mathrm{f}}$, the influence of $P_{\mathrm{sz}}$ is nonlinear as well, and the numerical value of $P_{\mathrm{sz}}$ can be not directly read from the ST data. Therefore, a best-match model calculation must be performed for analysis of the experimental data.

\section{Triple-layer PFP: Model}

The triple-layer PFP heterostructure renders a layered unipolar ferroelectric Bi-junction transistor with open base contact or double-diode arrangement, with common electrode anode and/or cathode combination depending on the external bias and combination of $P_{\mathrm{sz} 1}$ and $P_{\mathrm{sz} 2}$. As discussed below, the physical mechanisms within the polarization-coupled interfaces for the different schemes allow for interesting device applications. Figure 3 depicts model calculated ST data together with $w_{1}, w_{2}$ for a hypothetical $\mathrm{ZnO}$-BTO-ZnO heterostructure for the four polarization vector arrangements. Model parameters are those in Table II, except for $P_{\mathrm{sz}}$ $=0.2 \mu \mathrm{C} / \mathrm{cm}^{2}$. Note that for the calculations in Fig. $3 \mathrm{we}$ assumed $N_{\mathrm{c} 1}=N_{\mathrm{c} 2}=2 \times 10^{17} \mathrm{~cm}^{-3}$. The ST loops are shown for different sweeping voltages, and $w_{1}, w_{2}$ are shown for $V_{\mathrm{P}-\mathrm{P}}=12 \mathrm{~V}$ only. Panels in Figs. 3(a)-3(d) correspond to arrangements $\mathrm{P}^{-} \mathrm{FP}^{-}, \mathrm{P}^{+} \mathrm{FP}^{+}, \mathrm{P}^{-} \mathrm{FP}^{+}$, and $\mathrm{P}^{+} \mathrm{FP}^{-}$, respectively.

Arrangements $\mathrm{P}^{-} \mathrm{FP}^{-}$and $\mathrm{P}^{+} \mathrm{FP}^{+}$result in equivalent data as expected, which follow from simple physical contact inversion (voltage axis and abscissa inversion). For arrangements $\mathrm{P}^{-} \mathrm{FP}^{-}$and $\mathrm{P}^{+} \mathrm{FP}^{+}$the heterostructure remains within schemes (B) and (C), i.e., either one of $w_{1}$ or $w_{2}$ is zero or finite, and both depletion layers are neither zero nor finite at 
the same voltage. When the voltage sweeps from negative to positive, the heterostructure is represented by scheme (C), and $w_{1}$ is zero. When $w_{2}$ approaches zero, the heterostructure switches between operation schemes (C) to (B). For $\mathrm{P}^{-} \mathrm{FP}^{-}$ $\left(\mathrm{P}^{+} \mathrm{FP}^{+}\right)$the symmetric $P_{\mathrm{sz}}$ orientations produce positive (negative) charge at the right and negative (positive) charge at the left interface of layer F (Fig. 1). The depletion layer widths for ground and biased layer $P$ reach different values at maximum input voltage. As a result, the voltages at which the depletion layers disappear are not symmetric, and shift to negative (positive) values for $\mathrm{P}^{-} \mathrm{FP}^{-}\left(\mathrm{P}^{+} \mathrm{FP}^{+}\right)$. This asymmetric depletion layer formation causes asymmetric charging upon cyclic external voltage variation, which is reflected by downward (upward) shift of the ST loop for $\mathrm{P}^{-} \mathrm{FP}^{-}\left(\mathrm{P}^{+} \mathrm{FP}^{+}\right)$ with increasing $V_{\mathrm{P}-\mathrm{P}}$. In scheme (B) the heterostructure is composed of a series of diodes in "forward" (junction PF) "reverse" (junction FP) condition, and in (C) it resembles a series of diodes in "reverse"-"forward" condition (Table I). Brief biasing by short voltage pulses can set the bijunction heterostructure into (i) sequence "reverse"-"forward" or (ii) "forward"-"reverse". For $\mathrm{P}^{-} \mathrm{FP}^{-}\left(\mathrm{P}^{+} \mathrm{FP}^{+}\right)$, depending on the heterostructure parameters, a negative (positive) voltage region exists where both sequences (i) and (ii) can be found depending on $P_{\mathrm{d}}$. The double-interface polarization coupling revealed in this heterostructure provides a switchable doublediode structure, where at nonzero bias a bistable configuration can be obtained exploiting the switchable polarization of layer F.

Arrangements $\mathrm{P}^{-} \mathrm{FP}^{+}$and $\mathrm{P}^{+} \mathrm{FP}^{-}$result in $\mathrm{ST}$ data without loop shift and symmetric depletion layer formation. In addition to schemes (B) and (C), scheme (A) [sequence (iii) "forward"-"forward"] and (D) [sequence (iv) "reverse""reverse"] occur. Operation in scheme (A) occurs for $\mathrm{P}^{-} \mathrm{FP}^{+}$ for descending $V_{\text {in }}$ from approximately $0 \mathrm{~V}$ to $-2 \mathrm{~V}\left(w_{1}\right.$ disappears at approximately $0 \mathrm{~V}$ and $w_{2}$ remains zero until approximately $-2 \mathrm{~V}$ ) and for ascending $V_{\text {in }}$ from approximately 0 to $2 \mathrm{~V}$. For $\mathrm{P}^{+} \mathrm{FP}^{-}$operation in scheme (D) for descending $V_{\text {in }}$ from approximately 0.5 to $-3 \mathrm{~V}$ ( $w_{1}$ disappears at approximately $-2.5 \mathrm{~V}$ and $w_{2}$ opens at approximately $0.5 \mathrm{~V}$ ) and for ascending $V_{\text {in }}$ from approximately -0.5 to $3 \mathrm{~V}$, whereas scheme (A) does not occur. The asymmetric $P_{\mathrm{sz}}$ orientations produce symmetric negative charges at the right and left interface of layer F. As a result, the depletion layer widths for ground and biased layers $P$ reach symmetric values for $V_{\mathrm{in}}= \pm V_{\mathrm{P}-\mathrm{P}}$. This symmetric depletion layer formation causes symmetric capacitance values for positive and negative potential drops between the sample electrodes and thus results in a symmetric ST loop centered at zero output voltage. In principle, the bijunction device can be electrically switched between four possible diode-diode sequences. The choice of materials with properties $P_{\mathrm{sz}}, P_{\mathrm{d}}\left(P_{\mathrm{s}}, P_{\mathrm{r}}, E_{\mathrm{c}}\right)$ determines the voltage regions in which these situations can be obtained. Similar to the double-layer FP situation, due to the nonlinear functionality and hysteresis of $P_{\mathrm{d}}$ versus $E_{\mathrm{f}}$, the influence of $P_{\mathrm{sz} 1}, P_{\mathrm{sz} 2}$ is nonlinear as well, and the numerical value and orientation of $P_{\mathrm{sz} 1,2}$ cannot be directly read from the ST data. Therefore, a best-match model calculation must be performed for analysis of experimental data.

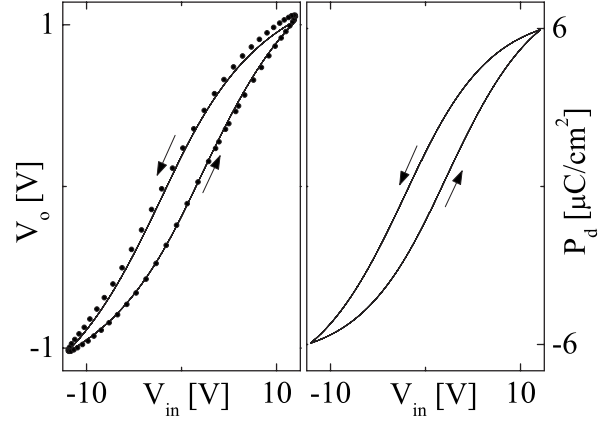

FIG. 4. Left panel: experimental (dotted line) and best-match model calculated ST data (solid line) from a single-layer F structure (Pt-BTO-Pt). Right panel: corresponding best-match model calculated ferroelectric polarization $P_{\mathrm{d}}$ within layer F (BTO). Best-match model parameters are given in Table II. Arrows indicate the input voltage sweep direction $\left(V_{\mathrm{P}-\mathrm{P}}=12 \mathrm{~V}, f=1.5 \mathrm{kHz}, P_{\mathrm{sz} 1}=P_{\mathrm{sz} 2}=0\right)$.

\section{Single-layer F: Experiment}

The left panel of Fig. 4 depicts experimental ST data and best-match model calculations for a single-layer F structure with ohmic metal contacts (Pt-BTO-Pt). The ST data reveal the typical ferroelectric hysteresis behavior. Domain formation within the polycrystalline film causes broadening (softening) of the ferroelectric switching behavior. This structure trivially operates in scheme (A), and the applied external potential is responsible for changes in $P_{\mathrm{d}}$ and internal electric field $E_{\mathrm{f}}$ within in layer F. Note that $P_{\mathrm{sz} 1}=P_{\mathrm{sz} 2}=0$. The right panel of Fig. 4 graphs the best-match model calculated trace of $P_{\mathrm{d}}$ versus applied voltage. The shape of the polarization loop is similar to the ST loop since all capacitance changes within the single-layer F structure are only caused by $P_{\mathrm{d}}$, the polarization of layer $\mathrm{F}$, which is directly proportional to the inverse capacitance of layer F. Slight deviations from the model and experimental ST data at positive voltages indicate formation of small accumulation layers at the interface of the Pt-top contact, which are not further considered here as their influence is marginal. The best-match model calculations revealed remanent polarization $P_{\mathrm{r}}$, saturation polarization $P_{\mathrm{s}}$, coercive field $E_{\mathrm{c}}$, and $\varepsilon_{\mathrm{f}} . d_{\mathrm{f}}$ was obtained from structural analysis. All parameters are summarized in Table II. Parameters obtained for $P_{\mathrm{r}}, E_{\mathrm{c}}$, and $\varepsilon_{\mathrm{f}}$ are within the range of previously reported data. ${ }^{68-70}$ The magnitude of $P_{\mathrm{s}}$ is about two to four times smaller than typical values reported from bulk single-crystal measurements. Smaller values are often observed in polycrystalline film samples, where full saturation may not be reached due to limitations in applicable voltages without irreversible formation of electrical breakthroughs. ${ }^{71-74}$

\section{E. Double-layer PF: Experiment}

Figure 5 depicts experimental and best-model calculated ST data together with the $P_{\mathrm{d}}$ variations in layer $\mathrm{F}$ for a double-layer PF structure and for different maximum sweeping voltages $V_{\mathrm{P}-\mathrm{P}}$. The best-match model parameters are given in Table II. Triangles indicate the transitions between schemes (A) and (B), indicative for depletion layer formation within the $\mathrm{ZnO}$ layer as predicted within our model scheme 


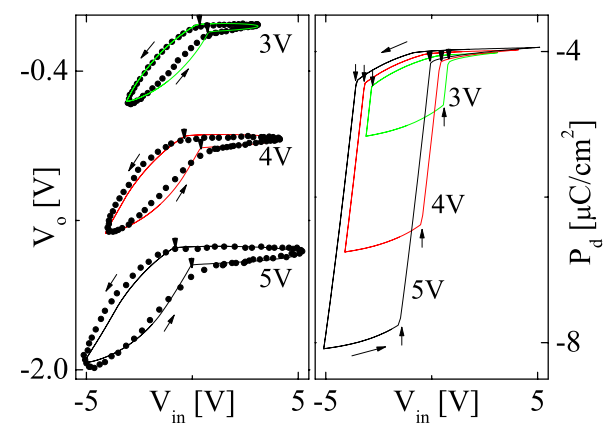

FIG. 5. (Color online) Same as Fig. 4 for the double-layer PF heterostructure (Pt-ZnO-BTO-Pt; $V_{\mathrm{P}-\mathrm{P}}=3,4$, and $5 \mathrm{~V} ; f=1.5 \mathrm{kHz}$; $\left.P_{\mathrm{sz}}=-4.1 \mu \mathrm{C} / \mathrm{cm}^{2}\right)$.

developed in Sec. II. Note that for this experiment full depletion condition, i.e., scheme $(\mathrm{E})$, is not reached. The ST data reflect the diodelike behavior of the overall heterostructure. The experimental data proof our suggested concept of interface polarization coupling. Both $\mathrm{ZnO}$ and BTO spontaneous polarizations determine the voltages of the transition points (triangles), which shift along the abscissa for increasing $V_{\mathrm{P}-\mathrm{P}}$. The separation between the transition points for "reverse" and "forward" directions are influenced by the ferroelectric hysteresis of the BTO layer. The separation is sensitive to model parameters $E_{\mathrm{c}}, P_{\mathrm{s}}$, and $P_{\mathrm{r}}$ for layer F. The right panel in Fig. 5 indicates ferroelectric polarization (downward arrows) and depolarization (upward arrows). On the ascending traces in Fig. 5, magnitude and orientation of $P_{\mathrm{sz}}$ are responsible for the voltage separation between transition (A)-(B) (right triangle on each loop, left panel) and depolarization transition (upward arrows, right panel). Transition (A)-(B) occurs prior to depolarization, thus $P_{\mathrm{sz}}$ must be negative. For $P_{\mathrm{sz}}=0$ both transitions would occur at the same input voltage. From our best-match model calculation we derive the spontaneous polarization of $\mathrm{ZnO}$ as $P_{\mathrm{sz}}=$ $-4.1 \mu \mathrm{C} \mathrm{cm}^{-2}$. This value matches very well with previous literature values $7 \pm 2 \mu \mathrm{C} \mathrm{cm}{ }^{-2} \cdot{ }^{24,30,31,75}$ The BTO coercive field $\left(E_{\mathrm{c}}=11 \mathrm{kV} / \mathrm{cm}\right)$ corresponds well to within typical values $(1.5$ to $50 \mathrm{kV} / \mathrm{cm})$ for BTO thin layers reported previously. ${ }^{68-70}$ Parameter $N_{\mathrm{c}}$ is in agreement with electrical Hall and infrared ellipsometry measurements. ${ }^{52}$ Due to the diodelike behavior of the heterostructure, different charge accumulation occurs at positive and negative bias. The center of gravity of the polarization loop remains negative. In "reverse" configuration smaller charge accumulation occurs and subsequently smaller ferroelectric polarization than in "forward" situation. Accordingly, the best-match calculated traces $P_{\mathrm{d}}$ reveal large negative polarization in scheme (A) and almost zero polarization in scheme (B), as discussed in Sec. IV B. For larger $V_{\mathrm{P}-\mathrm{P}}, P_{\mathrm{d}}$ in "forward" situation increases, causing further negative charging (downward shift of ST data), which is quantitatively described by our model approach. A similar voltage-dependent polarization drift was observed previously in MFS heterostructures and was qualitatively explained with asymmetric leakage currents. ${ }^{7-11}$

\section{F. Triple-layer PFP: Experiment}

Figure 6 depicts experimental and best-model calculated ST data together with the $P_{\mathrm{d}}$ variations in layer F for a triple-

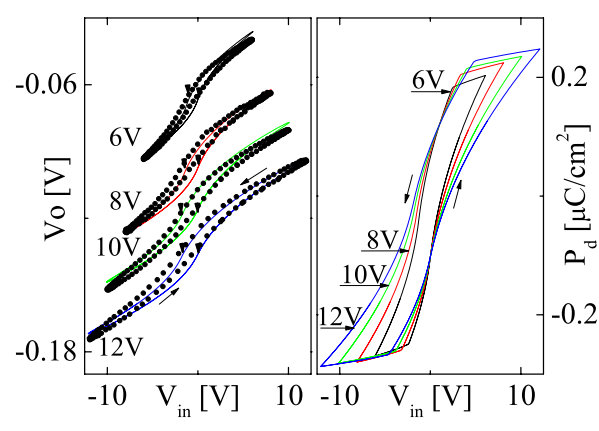

FIG. 6. (Color online) Same as Fig. 4 for the triple-layer PFP heterostructure (Pt-ZnO-BTO-ZnO-Pt; $V_{\mathrm{P}-\mathrm{P}}=6,8,10$, and $12 \mathrm{~V} ; f$ $=1.5 \mathrm{kHz}$ ).

layer PFP situation at various $V_{\mathrm{P}-\mathrm{P}}$. Similar to the doublelayer structure, best-model calculated and experimental data are in very good quantitative agreement. The best-match model parameters are summarized in Table II. From the analysis procedure we identify transitions between schemes (B) and (C) (triangles, left panel) where our sample switches between (i) sequence "reverse"-"forward" (on descending side of loop) and (ii) "forward"-"reverse" (on ascending side of loop). Note that the switching voltages are not symmetric with respect to zero bias, and are shifted to negative bias. Comparison with Fig. 3 identifies that our structure belongs to configuration $\mathrm{P}^{-} \mathrm{FP}^{-}$, i.e., both $\mathrm{ZnO}$ layers posses negatively oriented (toward the substrate) spontaneous polarization vector orientations. Note that calculations in Fig. 3 ideally assumed equivalent net donor concentration in both layers $\mathrm{P}$, whereas best-model calculations revealed slightly different concentrations within the $\mathrm{ZnO}$ layers (Table II; $N_{\mathrm{c} 1}$ and $N_{\mathrm{c} 2}$ are found in agreement with electrical Hall and infrared ellipsometry measurements). ${ }^{52}$ Because $N_{\mathrm{c} 1}>N_{\mathrm{c} 2}$, further asymmetric charge accumulation occurs, which causes an overall downward shift of the experimental ST data with respect to those in Fig. 3(a). From our best-match model analysis we identify a much smaller value of $P_{\mathrm{sz}}=$ $-0.1 \mu \mathrm{C} \mathrm{cm}^{-2}$ for this heterostructure than for the doublelayer structure discussed above. The latter finding may be explained by domain formation within the $\mathrm{ZnO}$ layers, in which neighboring domains may possess alternating polarization vector orientations. The heterostructure response is then affected by the average over multiple domain orientations.

As discussed in Sec. IV C, the triple-layer PFP heterostructure renders a unipolar bijunction transistor device, in which two diodes are connected in series. Note that this bijunction configuration does not require electrical contact to the base region and establishes a simple two-terminal device. Depending on $P_{\mathrm{d}}$, at certain voltages, the two diodes can be set into bistable situations. Brief biasing by short voltage pulses here switch the bijunction heterostructure into situations (i) or (ii) and in which the heterostructure static series resistance is slightly different, e.g., at a given "read" voltage of $1 \mathrm{~V}$. Bistable switching operation of this heterostructure was presented recently. ${ }^{59} \mathrm{~A}$ similar switching behavior was observed previously in metal-ferroelectric-metal heterostructures and explained by the occurrence of serial diode arrangements. Bouregba et al. demonstrated upon a single- 
layer dielectric continuum model calculations augmented by parallel circuits of diode-resistance pairs that such hysteresis offset can occur due to asymmetric resistance changes. In their series model, two pairs of antiparallel oriented diodes with two different resistances mimic the effect of different leakage currents within the ferroelectric layer over the metal interface barriers into the metal contacts. ${ }^{11}$ In our heterostructures, the diodelike behavior originates from the variation in the depletion layers and the ferroelectric polarization. ${ }^{59}$ The PFP heterostructures discussed in our work possess potential for new ferroelectric-semiconductor thin-film device applications.

\section{CONCLUSIONS}

We have presented and applied a model to explain and quantify the polarization response of interface polarization coupled piezoelectric-semiconductor ferroelectric heterostructures. Our model approach consist of the augmentation of depletion layer formation within a series layer capacitance calculation, which predicts the occurrence of asymmetric charging and polarization properties caused by the coupling between the switchable and nonswitchable polarization. Experimental investigations performed on single-layer BTO, double-layer ZnO-BTO, and triple-layer ZnO-BTO-ZnO heterostructures are in very good agreement with our theoretical model approach. Our model approach allows for the derivation of the magnitude and orientation of the spontaneous polarization of the piezoelectric constituent from experiment. We identify interface polarization coupled triple-layer $\mathrm{ZnO}$ BTO-ZnO heterostructures as two-terminal unipolar ferroelectric bijunction transistor for possible application in memory storage.

\section{ACKNOWLEDGMENTS}

We acknowledge support by the Deutsche Forschungsgemeinschaft within the Sonderforschunsgbereich SFB 762 "Functional Oxide Interfaces," the CoE at UNL, the J. A. Woollam Foundation, NSF-DMR MRSEC QSPIN Grant No. 0820521, NSF-DMR Grant No. 0907475, and NSF-DMR Grant No. 0922937.

\section{APPENDIX A: HYPERBOLIC TANGENT FUNCTION FERROELECTRIC POLARIZATION MODEL}

An electric field-dependent ferroelectric polarization model can be obtained from the following phenomenological equations: ${ }^{2}$

$$
P_{\text {sat }}^{+}\left(E_{\mathrm{f}}\right)=P_{\mathrm{s}} \tanh \left[\frac{E_{\mathrm{f}}-E_{\mathrm{c}}}{2 \delta}\right],
$$

where the plus sign in the superscript indicates the dipole saturation polarization $P_{\text {sat }}^{+}$on the lower branch of the saturated polarization hysteresis loop at electric field $E_{\mathrm{f}}$ after a given electric field increment. $E_{\mathrm{c}}$ denotes the coercive field. The quantity $\delta$ is obtained from

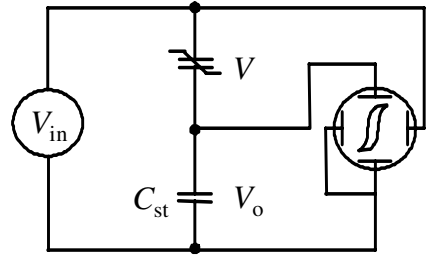

FIG. 7. Sawyer-Tower circuit.

$$
\delta=E_{\mathrm{c}}\left[\log \left(\frac{1+P_{\mathrm{r}} / P_{\mathrm{s}}}{1-P_{\mathrm{r}} / P_{\mathrm{s}}}\right)\right]^{-1},
$$

where $P_{\mathrm{r}}^{\prime}$ denotes the remanent polarization and $P_{\mathrm{s}}$ is the maximum saturation polarization. $P_{\text {sat }}^{-}$after a given electric field decrement is obtained from Eq. (A1) as follows:

$$
P_{\text {sat }}^{-}\left(E_{\mathrm{f}}\right)=-P_{\text {sat }}^{+}\left(-E_{\mathrm{f}}\right) \text {. }
$$

For an unsaturated polarization formation $P_{\mathrm{d}}$ does not necessarily follow the saturation hysteresis loop. In order to calculate an unsaturated hysteresis loop we used the approach suggested by Miller. ${ }^{3}$ In this model, the saturated and unsaturated polarizations at a given field $E_{\mathrm{f}}$ can be related by a factor $\Gamma$, which ensures smooth and monotonic approaching of the unsaturated polarization to the saturation polarization loop. This factor relates the first partial derivatives of both loops

$$
\frac{\partial P_{\mathrm{d}}}{\partial E_{\mathrm{f}}}=\Gamma \frac{\partial P_{\mathrm{sat}}}{\partial E_{\mathrm{f}}}
$$

and

$$
\Gamma=1-\tanh \left[\left(\frac{P_{\mathrm{d}}-P_{\mathrm{sat}}}{\xi P_{\mathrm{s}}-P_{\mathrm{d}}}\right)\right] .
$$

The factors $\xi=+1$ and $\xi=-1$ are for incrementing and decrementing fields $E_{\mathrm{f}}$, respectively, and $P_{\mathrm{d}}$ is the (ferroelectric) dipole polarization on the unsaturated hysteresis loop at $E_{\mathrm{f}}$, and $P_{\mathrm{d}}$ approaches $P_{\text {sat }}$ for saturated polarization.

\section{APPENDIX B: SAWYER-TOWER CIRCUIT MODEL}

Figure 7 shows the schematic of the Sawyer-Tower circuit. $C_{\mathrm{st}}$ is a normal (standard) test capacitor, and $V$ is the potential across a sample. $V_{\text {in }}$ denotes the potential applied to the series of both capacitances. $V_{\mathrm{o}}$ is the output voltage measured across the normal capacitor. $V_{\mathrm{bi}}$ is the effective built-in potential (sum of all conduction band offsets) across the sample. The time derivative of the charge within the circuitry is described following Miller et al. ${ }^{2}$

$$
\frac{d}{d t}\left(-\sigma_{\mathrm{b}} A_{\mathrm{f}}+C_{\mathrm{st}} V_{\mathrm{o}}\right)=\frac{V_{\mathrm{in}}-V_{\mathrm{o}}-V_{\mathrm{bi}}}{R_{\mathrm{s}}}-\frac{V_{\mathrm{o}}}{R_{\mathrm{n}}},
$$

where $A_{\mathrm{f}}$ is the contact area of the sample, $R_{\mathrm{s}}$ is the total (series) sample resistance between ground and bias electrode, and $R_{\mathrm{n}}$ is the normal capacitor resistance. With Eqs. (9) and (10), a differential relation is obtained for the PFP structure, which maps characteristic sample properties through experimental variation in $V_{\text {in }}$ and measurement of $V_{\mathrm{o}}$ : 
TABLE III. $w_{1_{l}}=\left(-b \pm \sqrt{b^{2}-4 a c}\right) /(2 a)>0$, after iteration step $(l-1)$.

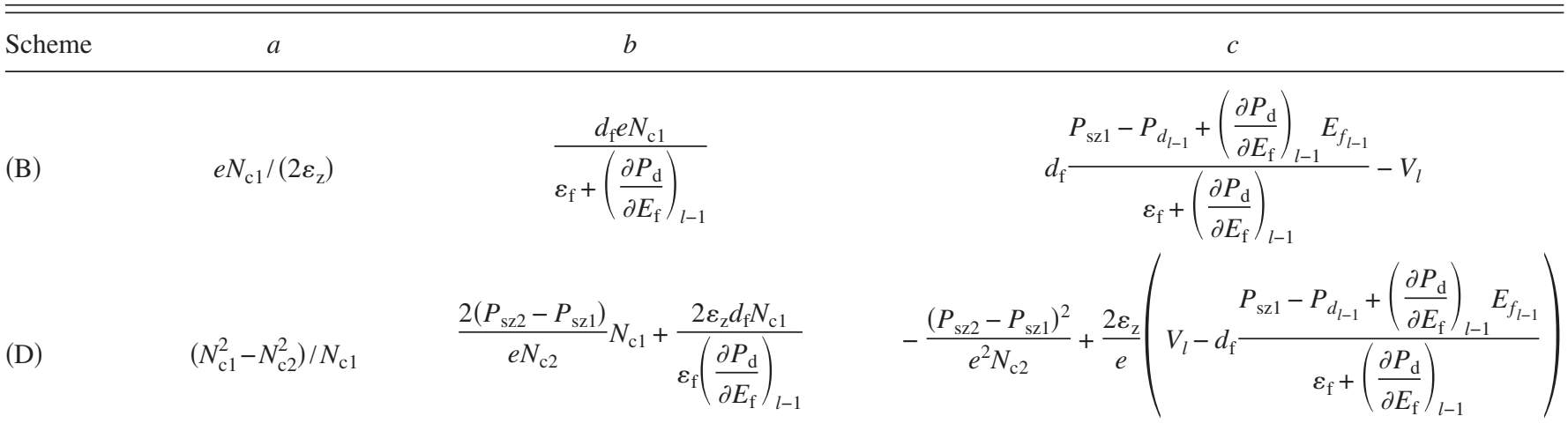

$$
\frac{d V_{\mathrm{o}}}{d t}=\frac{\frac{V_{\mathrm{in}}-V_{\mathrm{o}}-V_{\mathrm{bi}}}{R_{\mathrm{s}}}-\frac{V_{\mathrm{o}}}{R_{\mathrm{n}}}+\gamma\left(\varepsilon_{\mathrm{f}}+\frac{d P_{\mathrm{d}}}{d E_{\mathrm{f}}}\right) A_{\mathrm{f}} \frac{d V_{\mathrm{in}}}{d t}}{C_{\mathrm{st}}+\gamma\left(\varepsilon_{\mathrm{f}}+\frac{d P_{\mathrm{d}}}{d E_{\mathrm{f}}}\right) A_{\mathrm{f}}} .
$$

ST data represent the ratio of the inverse standard capacitance $C_{\mathrm{st}}^{-1}$ versus the sum of sample $C_{\mathrm{s}}^{-1}$ and standard $C_{\mathrm{st}}^{-1}$ inverse capacitances. The slope of the ST data is bound between $1\left(C_{\mathrm{s}} \rightarrow 0\right)$ and $0\left(C_{\mathrm{s}} \rightarrow \infty\right)$. For ideally nonconductive sample structures, a differential increase or decrease in the ST data slope at a given input voltage represents a decrease or increase in the sample capacitance, respectively.

\section{APPENDIX C: ITERATION ALGORITHM}

Equation (B2) is solved by iteration, which is adopted from Miller et $a .^{2}$ In this algorithm, external voltage loops are iterated, and at each voltage $P_{\mathrm{d}}, E_{\mathrm{f}}, w_{1}$, and $w_{2}$ are searched for iteratively, until convergence is obtained due to the nonlinear dependence between $P_{\mathrm{d}}$ and $E_{\mathrm{f}}$. Convergence is reached when repeated loop calculations produce stable identical loops. At given external potential $V_{l}$, after $l-1$ iterations, $P_{\mathrm{d}_{l}}$ is calculated from

$$
P_{\mathrm{d}_{l}}=P_{\mathrm{d}_{l-1}}+\left(\frac{\partial P_{\mathrm{d}}}{\partial E_{\mathrm{f}}}\right)_{l-1}\left(E_{\mathrm{f}_{l}}-E_{\mathrm{f}_{l-1}}\right) .
$$

For calculation of $E_{\mathrm{f}}$ and subsequently $\left(\frac{\partial P_{\mathrm{d}}}{\partial E_{\mathrm{f}}}\right)$, evaluation of $w_{1}, w_{2_{l}}$ is necessary. From Tables III and IV one obtains solutions for $w_{1_{l}}$ and $w_{2}$, respectively. The iterative scheme evaluates first $w_{1}, w_{2}$, according to scheme in step $l-1$, and polarization and electric field in step $l-1$. Inspection of $w_{1}$, $w_{2}$ then determines if the current scheme (in step $l-1$ ) in Tables I, III, and IV is still valid in step $l$, or if it must be changed, i.e., if one and/or both depletion layers are zero, nonzero, or fully depleted. $E_{\mathrm{f}}$ is then calculated using the boundary conditions in Eqs. (5) and (6) for the appropriate scheme. No boundary exists in scheme (A), and we use Eq. (2)

$$
E_{\mathrm{f}_{l}}=\frac{V_{l}}{d_{\mathrm{f}}} .
$$

Equation (5) or Eq. (6) is valid for schemes (B) and (E) or (C) and (F), respectively,

$$
E_{\mathrm{f}_{l}}=\frac{ \pm e N_{\mathrm{c} 1,2} \omega_{1,2}+P_{\mathrm{sz} 1,2}-P_{\mathrm{d}_{l}}+\left(\frac{\partial P_{\mathrm{d}}}{\partial E_{\mathrm{f}}}\right)_{l} E_{\mathrm{f}_{l-1}}}{\varepsilon_{\mathrm{f}}+\left(\frac{\partial P_{\mathrm{d}}}{\partial E_{\mathrm{f}}}\right)_{l}}
$$

where the plus sign applies for (B) and (E), and the minus sign applies for $(\mathrm{C})$ and $(\mathrm{F})$. The sum of both boundary conditions is considered for the remaining schemes (D), (G), $(\mathrm{H})$, and (I) in Table I

TABLE IV. $w_{2_{l}}=\left(-b \pm \sqrt{b^{2}-4 a c}\right) /(2 a)>0$, after iteration step $(l-1)$.

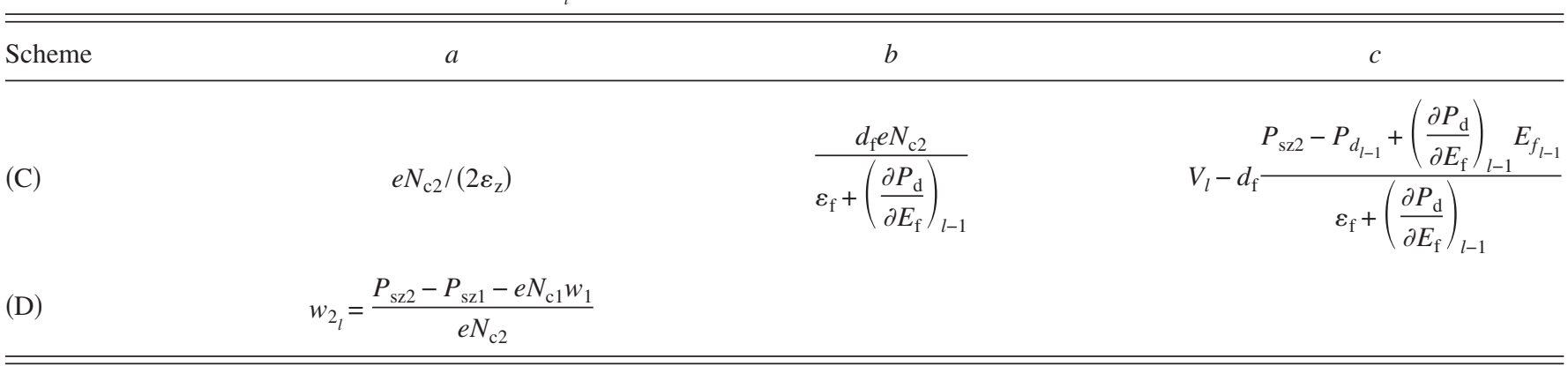




$$
E_{\mathrm{f}_{l}}=\frac{\frac{1}{2} e\left[N_{\mathrm{c} 1} \omega_{1_{l}}-N_{\mathrm{c} 2} \omega_{2_{l}}+P_{\mathrm{sz} 1}+P_{\mathrm{sz} 2}\right]-P_{\mathrm{d}_{l}}+\left(\frac{\partial P_{\mathrm{d}}}{\partial E_{\mathrm{f}}}\right)_{l} E_{\mathrm{f}_{l-1}}}{\varepsilon_{\mathrm{f}}+\left(\frac{\partial P_{\mathrm{d}}}{\partial E_{\mathrm{f}}}\right)_{l}} .
$$

Note that $w_{1_{l}}$ in (B), (D), (G) and $w_{2_{l}}$ in (C), (D), (H) are obtained as the physical meaningful (positive) branch of the quadratic equation following from Eq. (4), while $w_{1_{l}}=d_{\mathrm{z} 1}$ in (E), (H), (I) and $w_{2_{l}}=d_{\mathrm{z} 2}$ in (F), (G), (I).
${ }^{1}$ K. M. Rabe, C. H. Ahn, and J.-M. Triscone, Physics of Ferroelectrics: A Modern Perspective (Springer, New York, 2007).

${ }^{2}$ S. L. Miller, R. D. Nasby, J. R. Schwank, M. S. Rodgers, and P. V. Dressendorfer, J. Appl. Phys. 68, 6463 (1990).

${ }^{3}$ S. L. Miller, J. R. Schwank, R. D. Nasby, and M. S. Rodgers, J. Appl. Phys. 70, 2849 (1991)

${ }^{4}$ C. H. Tsang, C. K. Wong, and F. G. Shin, J. Appl. Phys. 98, 084103 (2005).

${ }^{5}$ Z. Ye, M. H. Tang, C. P. Cheng, Y. C. Zhou, X. J. Zheng, and Z. S. Hu, J. Appl. Phys. 100, 094101 (2006).

${ }^{6}$ A. N. Morozovska, E. A. Eliseev, D. Remiens, and C. Soyer, J. Appl. Phys. 100, 014109 (2006).

${ }^{7}$ C. K. Wong and F. G. Shin, J. Appl. Phys. 98, 024104 (2005).

${ }^{8}$ C. K. Wong and F. G. Shin, Appl. Phys. Lett. 86, 042901 (2005).

${ }^{9}$ G. Poullain, R. Bouregba, B. Vilquin, G. L. Rhun, and H. Murray, Appl. Phys. Lett. 81, 5015 (2002).

${ }^{10}$ L. Zheng, C. Lin, W.-P. Xu, and M. Okuyama, J. Appl. Phys. 79, 8634 (1996).

${ }^{11}$ R. Bouregba, G. Poullain, B. Vilquin, and G. L. Rhun, J. Appl. Phys. 93, 5583 (2003).

${ }^{12}$ M. Schubert, N. Ashkenov, T. Hofmann, M. Lorenz, H. Hochmuth, H. V. Wenckstern, M. Grundmann, and G. Wagner, Ann. Phys. 13, 61 (2004).

${ }^{13}$ S.-Y. Wu, IEEE Trans. Electron Devices 21, 499 (1974).

${ }^{14}$ M. Brandt, H. Frenzel, H. Hochmuth, M. Lorenz, M. Grundmann, and J. Schubert, J. Vac. Sci. Technol. B 27, 1789 (2009).

${ }^{15}$ Y. Xu, C. J. Chen, R. Xu, and J. D. Mackenzie, J. Appl. Phys. 67, 2985 (1990).

${ }^{16}$ L. S. Berman, Semiconductors 39, 313 (2005).

${ }^{17}$ P. Wurfel and I. P. Batra, Phys. Rev. B 8, 5126 (1973).

${ }^{18}$ P. Wurfel and I. P. Batra, Phys. Rev. B 10, 1118 (1974).

${ }^{19}$ I. P. Batra, P. Wurfel, and B. D. Silverman, Phys. Rev. B 8, 3257 (1973).

${ }^{20}$ L. S. Berman, Semiconductors 35, 193 (2001).

${ }^{21}$ G. Z. Liu, K. J. Jin, J. Qiu, M. He, H. B. Lu, J. Xing, Y. L. Zhou, and G. Z. Yang, Appl. Phys. Lett. 91, 252110 (2007).

${ }^{22}$ E. Cagin, D. Y. Chen, J. J. Siddiqui, and J. D. Phillips, J. Phys. D 40, 2430 (2007).

${ }^{23}$ A. Malashevich and D. Vanderbilt, Phys. Rev. B 75, 045106 (2007).

${ }^{24}$ A. Dal Corso, M. Posternak, R. Resta, and A. Baldereschi, Phys. Rev. B 50, 10715 (1994).

${ }^{25}$ Y. Noel, C. M. Zicovich-Wilson, B. Civalleri, P. D'Arco, and R. Dovesi, Phys. Rev. B 65, 014111 (2001).

${ }^{26}$ O. Ambacher et al., J. Appl. Phys. 85, 3222 (1999).

${ }^{27}$ O. Ambacher et al., J. Appl. Phys. 87, 334 (2000).

${ }^{28}$ M. Dawber, K. M. Rabe, and J. F. Scott, Rev. Mod. Phys. 77, 1083 (2005).
${ }^{29}$ P. Gopal and N. Spaldin, J. Electron. Mater. 35, 538 (2006).

${ }^{30}$ Y.-I. Kim, K. Page, and R. Seshadri, Appl. Phys. Lett. 90, 101904 (2007).

${ }^{31}$ J. Jerphagnon and H. W. Newkirk, Appl. Phys. Lett. 18, 245 (1971).

${ }^{32}$ S. Lautenschlaeger, J. Sann, N. Volbers, B. K. Meyer, A. Hoffmann, U. Haboeck, and M. R. Wagner, Phys. Rev. B 77, 144108 (2008).

${ }^{33}$ M. W. Allen, S. M. Durbin, and J. B. Metson, Appl. Phys. Lett. 91, 053512 (2007).

${ }^{34}$ M. W. Allen, P. Miller, R. J. Reeves, and S. M. Durbin, Appl. Phys. Lett. 90, 062104 (2007).

${ }^{35}$ Y. Dong, Z.-Q. Fang, D. C. Look, G. Cantwell, J. Zhang, J. J. Song, and L. J. Brillson, Appl. Phys. Lett. 93, 072111 (2008).

${ }^{36}$ R. Y. Gunji, M. Nakano, A. Tsukazaki, A. Ohtomo, T. Fukumura, and M. Kawasaki, Appl. Phys. Lett. 93, 012104 (2008).

${ }^{37}$ D. Kohl, M. Henzler, and G. Heiland, Surf. Sci. 41, 403 (1974).

${ }^{38}$ O. Dulub, U. Diebold, and G. Kresse, Phys. Rev. Lett. 90, 016102 (2003)

${ }^{39}$ G. Heiland, P. Kunstmann, and H. Pfister, Z. Phys. A: Hadrons Nucl. 176, 485 (1963).

${ }^{40}$ H. Tampo, P. Fons, A. Yamada, K.-K. Kim, H. Shibata, K. Matsubara, S. Niki, H. Yoshikawa, and H. Kanie, Appl. Phys. Lett. 87, 141904 (2005).

${ }^{41}$ R. L. Barns, E. T. Keve, and S. C. Abrahams, J. Appl. Crystallogr. 3, 27 (1970).

${ }^{42}$ A. N. Mariano and R. E. Hanneman, J. Appl. Phys. 34, 384 (1963).

${ }^{43}$ S. Kazuta, Y. Cho, H. Odagawa, and M. Kadota, Jpn. J. Appl. Phys. 39, 3121 (2000).

${ }^{44}$ M. Sambi, G. Granozzi, G. A. Rizzi, M. Casarin, and E. Tondello, Surf. Sci. 319, 149 (1994).

${ }^{45}$ M. Galeotti, A. Atrei, U. Bardi, G. Rovida, M. Torrini, E. Zanazzi, A. Santucci, and A. Klimov, Chem. Phys. Lett. 222, 349 (1994).

${ }^{46}$ T. Ohnishi, A. Ohtomo, I. Ohkuboa, M. Kawasaki, M. Yoshimoto, and H. Koinuma, Mater. Sci. Eng., B 56, 256 (1998).

${ }^{47}$ S.-K. Hong, T. Hanada, H.-J. Ko, Y. Chen, T. Yao, D. Imai, K. Araki, and M. Shinohara, Appl. Phys. Lett. 77, 3571 (2000).

${ }^{48}$ I. Ohkubo, A. Ohtomo, T. Ohnishi, Y. Mastumoto, H. Koinuma, and M. Kawasaki, Surf. Sci. 443, L1043 (1999).

${ }^{49}$ M. Yano, K. Hashimoto, K. Fujimoto, K. Koike, S. Sasa, M. Inoue, Y. Uetsuji, T. Ohnishi, and K. Inaba, J. Cryst. Growth 301-302, 353 (2007).

${ }^{50}$ A. Tsukazaki, A. Ohtomo, T. Kita, Y. Ohno, H. Ohno, and M. Kawasaki, Science 315, 1388 (2007).

${ }^{51}$ H. Tampo et al., Appl. Phys. Lett. 93, 202104 (2008). 
${ }^{52}$ C. Bundesmann, R. Schmidt-Grund, and M. Schubert, Transparent Conductive Zinc Oxide, Springer Series in Material Science, Vol. 104 (Springer, Berlin, 2007), Chap. Optical Properties of $\mathrm{ZnO}$ and Related Compounds.

${ }^{53}$ T. Bretagnon, P. Lefebvre, T. Guillet, T. Taliercio, B. Gil, and C. Morhain, Appl. Phys. Lett. 90, 201912 (2007).

${ }^{54}$ B. N. Mbenkum, N. Ashkenov, M. Schubert, M. Lorenz, H. Hochmuth, D. Michel, M. Grundmann, and G. Wagner, Appl. Phys. Lett. 86, 091904 (2005).

${ }^{55}$ V. M. Voora, T. Hofmann, M. Brandt, M. Lorenz, M. Grundmann, and M. Schubert, Phys. Status Solidi C 5, 1328 (2008).

${ }^{56}$ N. Ashkenov, M. Schubert, E. Twerdowski, H. v. Wenckstern, B. Mbenkum, H. Hochmuth, M. Lorenz, W. Grill, and M. Grundmann, Thin Solid Films 486, 153 (2005).

${ }^{57}$ V. M. Voora, T. Hofmann, M. Brandt, M. Lorenz, M. Grundmann, N. Ashkenov, and M. Schubert, J. Electron. Mater. 37, 1029 (2008).

${ }^{58}$ V. M. Voora, T. Hofmann, M. Brandt, M. Lorenz, M. Grundmann, N. Ashkenov, and M. Schubert, Appl. Phys. Lett. 94, 142904 (2009).

${ }^{59}$ V. M. Voora, T. Hofmann, M. Brandt, M. Lorenz, N. Ashkenov, M. Grundmann, and M. Schubert, Appl. Phys. Lett. 95, 082902 (2009).

${ }^{60}$ V. M. Voora, T. Hofmann, M. Brandt, M. Lorenz, M. Grundmann, and M. Schubert, in Theory and Applications of Ferroelectric and Multiferroic Materials, edited by M. Dawber, MRS Symposia Proceedings No. 1110E (Material Research Society, Warrendale, PA, 2009), p. 1110-C06-14.

${ }^{61}$ A. L. Roytburd, S. Zhong, and S. P. Alpay, Appl. Phys. Lett. 87, 092902 (2005).
${ }^{62}$ S. M. Nakhmanson, K. M. Rabe, and D. Vanderbilt, Appl. Phys. Lett. 87, 102906 (2005).

${ }^{63}$ M. B. Okatan, M. W. Cole, and S. P. Alpay, J. Appl. Phys. 104, 104107 (2008).

${ }^{64}$ A. M. Bratkovsky and A. P. Levanyuk, Phys. Rev. B 61, 15042 (2000).

${ }^{65}$ M. B. Okatan, J. V. Mantese, and S. P. Alpay, Phys. Rev. B 79, 174113 (2009).

${ }^{66}$ A. M. Bratkovsky and A. P. Levanyuk, Phys. Rev. Lett. 86, 3642 (2001).

${ }^{67}$ E. D. Murray and D. Vanderbilt, Phys. Rev. B 79, 100102(R) (2009).

${ }^{68}$ M. E. Line and A. M. Glass, Principle and Applications of Ferroelectrics and Related Materials (Clarendon, Oxford, 1977).

${ }^{69}$ A. Li, C. Ge, P. Lü, D. Wu, S. Xiong, and N. Ming, Appl. Phys. Lett. 70, 1616 (1997).

${ }^{70}$ W. J. Lin, T. Y. Tseng, H. B. Lu, S. L. Tu, S. J. Yang, and I. N. Lin, J. Appl. Phys. 77, 6466 (1995).

${ }^{71}$ B. A. Strukov, S. T. Davitadze, S. N. Kravchun, S. A. Taraskin, M. Goltzman, V. V. Lemanov, and S. G. Shulman, J. Phys.: Condens. Matter 15, 4331 (2003).

${ }^{72}$ H. Miyazawa, E. Natori, T. Shimoda, H. Kishimoto, F. Ishii, and T. Oguchi, Jpn. J. Appl. Phys. 40, 5809 (2001).

${ }^{73}$ T. Mitsui, I. Tatsuzaki, and E. Nakamura, An Introduction to the Physics of Ferroelectrics (GBSP, Inc., New York, 1976).

${ }^{74}$ D. Fu, M. Itoh, and S. Y. Koshihara, J. Phys.: Condens. Matter 22, 052204 (2010).

${ }^{75}$ F. Bernardini, V. Fiorentini, and D. Vanderbilt, Phys. Rev. B 56, R10024 (1997). 